\title{
Validity of Recreational Marathon Runners' Self-Reported Anthropometric Data
}

\author{
Nikolaidis, Pantelis T ; Knechtle, Beat
}

\begin{abstract}
While studies on large samples of recreational runners have often relied on participants' self-reported height and body mass, the validity of these data have not been investigated for this population. Hence, this study sought to examine the validity of self-reported anthropometric measures among recreational marathon runners. Female $(\mathrm{n}=32)$ and male $(\mathrm{n}=135)$ recreational marathon runners were requested to estimate their body mass and height (and we calculated their self-reported body mass index [BMI]), after which we took actual measures of their body mass and height and calculated their actual BMI. Participants' self-reported values underestimated their actual body mass by $0.65 \mathrm{~kg}(\mathrm{p}<.001, \eta 2=0.222)$ and their actual BMI by $0.35 \mathrm{~kg} \cdot \mathrm{m}-2(\mathrm{p}<.001, \eta 2=0.245)$. There was a significant Assessment Method $\times$ Sex Interaction for both body mass $(\mathrm{p}=.019, \eta 2=0.033)$ and BMI $(\mathrm{p}=.017, \eta 2=0.034)$, as women underestimated body mass values more than men. Participants overestimated their height by $0.44 \mathrm{~cm}(\mathrm{p}<.001, \eta 2=0.075)$, but the interaction of sex and assessment method for height was not statistically significant. Underestimates of body mass correlated with marathon racing speed $(r=.24, p=.006)$ and body fat percentage $(r=-.29, p=.001)$ in men, but not in women $(p>.05)$. The disagreement between selfreported and measured anthropometric data in the present sample was lower than has been previously reported for the general population, suggesting that marathon runners may more accurately self-perceive and/or report their anthropometric characteristics. These findings are of practical value for health professionals and researchers (e.g., nutritionists and exercise physiologists) questionnaires to recreational marathon runners.
\end{abstract}

DOI: https://doi.org/10.1177/0031512520930159

Posted at the Zurich Open Repository and Archive, University of Zurich

ZORA URL: https://doi.org/10.5167/uzh-188133

Journal Article

Accepted Version

Originally published at:

Nikolaidis, Pantelis T; Knechtle, Beat (2020). Validity of Recreational Marathon Runners' Self-Reported Anthropometric Data. Perceptual and Motor Skills, 127(6):1068-1078.

DOI: https://doi.org/10.1177/0031512520930159 
4

\title{
Validity of Recreational Marathon Runners' Self-Reported Anthropometric Data
}

\author{
Pantelis T. Nikolaidis ${ }^{1,2}$, Beat Knechtle ${ }^{3,4}$ \\ ${ }^{1}$ School of Health and Caring Sciences, University of West Attica, Egaleo, Greece \\ ${ }^{2}$ Exercise Physiology Laboratory, Nikaia, Greece \\ 3 Institute of Primary Care, University of Zurich, Zurich, Switzerland; \\ ${ }^{4}$ Medbase St. Gallen Am Vadianplatz, St. Gallen, Switzerland
}

Medbase St. Gallen Am Vadianplatz, St. Gallen, Switzerland

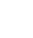

(1)

(6)

(1)

(1)

(1)

(1)

\section{$(5$}

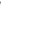

(1)

0

\section{2} (3)

\section{Correspondence}

Prof. Dr. med. Beat Knechtle

Facharzt FMH fürAllgemeinmedizin

Medbase St. Gallen Am Vadianplatz

Vadianstrasse 26

9001 St. Gallen, Switzerland

Telefon / Telefax: +41 (0) 712269300 / +41 (0) 712269301

E-mail: beat.knechtle@hispeed.ch 
44 While studies on large samples of recreational runners have often relied on participants' self45 reported height and body mass, the validity of these data have not been investigated for this 46 population. Hence, this study sought to examine the validity of self-reported anthropometric 47 measures among recreational marathon runners. Female $(n=32)$ and male $(n=135)$ 48 recreational marathon runners were requested to estimate their body mass and height (and we 49 calculated their 'self-reported' body mass index [BMI]), after which we took actual measures 50 of their body mass and height and calculated their actual BMI. Participants' self-reported 51 values underestimated their actual body mass by $0.65 \mathrm{~kg}\left(p<0.001, \eta^{2}=0.222\right)$ and their actual BMI by $0.35 \mathrm{~kg} \cdot \mathrm{m}^{-2}\left(p<0.001, \eta^{2}=0.245\right)$. There was a significant assessment method $\times$ sex 53 interaction for both body mass $\left(p=0.019, \eta^{2}=0.033\right)$ and BMI $\left(p=0.017, \eta^{2}=0.034\right)$, as women 54 underestimated body mass values more than men. Participants' overestimated their height by $550.44 \mathrm{~cm}\left(p<0.001, \eta^{2}=0.075\right)$, but the interaction of sex and assessment method for height 56 was not statistically significant. Underestimates of body mass correlated with marathon 57 racing speed $(r=0.24, p=0.006)$ and body fat percentage $(r=-0.29, p=0.001)$ in men, but not in women $(p>0.05)$. The disagreement between self-reported and measured anthropometric 59 data in the present sample was lower than has been previously reported for the general 60 population, suggesting that marathon runners may more accurately self-perceive and/or 61 report their anthropometric characteristics. These findings are of practical value for health 62 professionals and researchers (e.g. nutritionists, exercise physiologists) questionnaires to 63 recreational marathon runners.

64 Keywords: anthropometry; endurance exercise; measurement error; questionnaire 
The number of annual marathon races and participant finishers has continuously

67

increased in recent years due to a growing number of recreational runners (Vitti, Nikolaidis, Villiger, Onywera, \& Knechtle, 2019). Scientific research on recreational marathon runners has often relied on questionnaires using self-reported measures of body mass, height and even body mass index (BMI) (Boldt et al., 2018; Ponzio et al., 2018; Vickers \& Vertosick, 2016). While practical, this low cost, large sample data collection method is subject to response bias (Connor Gorber, Tremblay, Moher, \& Gorber, 2007), leading Seigjo et al. (2018) to identify a need to evaluate the accuracy of self-reported anthropometric data.

Most existing studies of self-reported anthropometric data were conducted on general population samples, with little data available for athlete samples (Knechtle, Rust, Rosemann, Knechtle, \& Bescos, 2012), particularly recreational marathon runners. BMI has been related to both sport performance (race time) (Vickers \& Vertosick, 2016) and prevalence of injuries (Vitez, Zupet, Zadnik, \& Drobnic, 2017) in marathon runners who share qualities with both athletes and the general population. Despite its uncertain accuracy, self-reported data on height, body mass and BMI have been widely used in research regarding marathon runners (Boldt et al., 2018; Holmich, Christensen, Darre, Jahnsen, \& Hartvig, 1989; Ponzio et al., 2018; Vadeboncoeur et al., 2012; Vickers \& Vertosick, 2016; Vitez et al., 2017). Understanding the accuracy of these data is of importance to both researchers and practitioners. Moreover, considering the increased number of women and older competitors in marathon races, the relation between sex and age and anthropometric data accuracy should be examined. Consequently, the objectives of this study were to investigate differences between self-reported and measured height, body mass and BMI among recreational marathon runners, while also examining any interactions between this accuracy and participant sex and age. We hypothesized that marathon runners would over-report height 
90 and under-report body mass resulting in a lower BMI value (Nieto-García, Bush, \& Keyl,

91 1990; Seijo et al., 2018). Moreover, we assumed that the bias in self-reporting anthropometric

92 characteristics in recreational marathon runners would be smaller than that observed among

93 the general population, considering the specific physiological and psychological

94 characteristics of athletes (Knechtle et al., 2012). For the purpose of this study, 'self-reported

95 BMI' referred to BMI calculated from self-reported height and body mass. 


\section{Participants}

Participants were 32 female $(M$ age $=44.3, S D=8.7$ years; $M$ height $=176.2, S D=5.9$ $\mathrm{cm} ; M$ body mass $=57.7, S D=7.5 \mathrm{~kg} ;$ and $\left.M \mathrm{BMI}=21.8, S D=2.2 \mathrm{~kg} \cdot \mathrm{m}^{-2}\right)$ and 135 male $(M$ age $=40.1, S D=9.0$ years; $M=$ height $162.3, S D=6.5 \mathrm{~cm} ; M$ body mass $=76.9, S D=9.3 \mathrm{~kg}$; and $M=\mathrm{BMI}=24.8, S D=2.6 \mathrm{~kg} \cdot \mathrm{m}^{-2}$ ) recreational marathon runners. Participants were invited through local media and sport clubs in the Attica region of Greece. The only inclusion criterion was participating and finishing the 2017 Athens marathon. After having been informed about the research procedures, all participants provided written informed consent. The study design was approved by the Exercise Physiology Laboratory, Nikaia, Greece (EPL 2017/7) in accordance with the Declaration of Helsinki revised in 2013.

\section{Procedures}

The present study was part of a larger project on physiological characteristics of recreational marathon runners for which detailed procedures were published in Nikolaidis, Chalabaev, Rosemann and Knechtle (2019) and Nikolaidis, Rosemann and Knechtle (2018a; 2018b). Briefly, in the context of the participants' visit to an exercise physiology laboratory to perform a series of anthropometric and exercise tests, participants were administered a questionnaire including items about their height and body mass. It should be highlighted that participants were aware when completing the questionnaire that they would later have their, height and body mass measured. Based on their self-reported height and body mass index, we calculated their 'self-reported' BMI. Height and body mass were then actually measured with participants barefoot and wearing minimal clothing. We used a weighing scale (Tanita, Arlington Heights, IL, USA) to measure body mass to the nearest $0.1 \mathrm{~kg}$, and a portable stadiometer (SECA Leicester, UK) to measure height to the nearest $0.1 \mathrm{~cm}$. BMI was 
120 calculated as the quotient of body mass $(\mathrm{kg})$ to height squared $\left(\mathrm{m}^{2}\right)$. A comparison of normal-

121 weight versus over-weight BMI groups was performed for male participants since there were

122 too few over-weight female participants $(n=1)$ to permit this comparison.

123 Statistical and data analysis

124 Statistical analyses were conducted using GraphPad Prism v. 7.0 (GraphPad Software, 125 San Diego, USA) and IBM SPSS v.23.0 (SPSS, Chicago, USA). We set statistical 126 significance at $p=0.05$. We tested the data distribution for normality using the Kolmogorov127 Smirnov test and visual inspecting Q-Q plots. Data are presented as means and standard 128 deviations. We used a between-within analysis of variance (ANOVA) to examine the main 129 and interaction effects of assessment method (self-reported and measured) and sex on height, 130 body mass and BMI, and we used eta squared $\left(\eta^{2}\right)$ to estimate the magnitude of these 131 differences (Cohen, 1988). We used Bland-Altman plots to analyze the agreement between 132 self-reported and measured values (Bland \& Altman, 1986), and we used Pearson correlation 133 coefficient $r$ to examine the relationship between the difference value of the reported minus 134 the measured and the average of the reported and measured value ((reported + measured $) / 2$ ) 135 for each anthropometric measure. In addition, we used $r$ to examine the relationship between 136 the self-report bias in anthropometric measures and age. We used an independent Student $t$ 137 test to compare the self-reported and measured height, body mass and BMI between normal138 weight and over-weight male participants, and we used Cohen's $d$ to evaluate the magnitude 139 of differences (Cohen, 1988). 
Participants' self-reported body mass significantly underestimated both their actual body

142 mass by an average of $0.65 \mathrm{~kg}\left(\mathrm{t}_{166}=6.762, p<0.001, \mathrm{~d}=0.06\right)$ and their actual BMI by an

143 average of $0.35 \mathrm{~kg} \cdot \mathrm{m}^{-2}\left(\mathrm{t}_{166}=7.288, p<0.001, \mathrm{~d}=0.13\right)$ (see Figure 1). An ANOVA also

144 revealed a significant assessment method $\times$ sex interaction for both body mass $\left(\mathrm{F}_{1,165}=5.576\right.$,

$\left.145 p=0.019, \quad \eta^{2}=0.033\right)$ and $\mathrm{BMI} \quad\left(\mathrm{F}_{1,165}=5.862, \quad p=0.017, \quad \eta^{2}=0.034\right), \quad$ with women

146 underestimating body mass and BMI more than men. Participants' self-reported height

147 significantly overestimated actual height by an average of $0.44 \mathrm{~cm}\left(\mathrm{t}_{166}=4.087, p<0.001\right.$,

$148 \mathrm{~d}=0.05$ ). There was no significant assessment method $\times$ sex interaction for height.

[Insert Figure 1 about here.]

150 The participant self-report bias (i.e., difference between participants' reported and

151 measured values) can be seen in Figure 2. There were statistically non-significant correlations

152 between this bias or difference between reported and measured values and the average of

153 these values $(($ reported + measured $) / 2$ ) for height (females, $r=-0.04$; males, $r=-0.13)$, body

154 mass (females, $r=-0.15$; males, $r=-0.14)$ and for females' BMI $(r=-0.17)$ while there was a

155 small but significant negative correlation between these variables for males' BMI $(r=-0.20$,

$156 p=0.019)$, indicating that males with higher measured BMI under-reported their BMI more

157 than did males with lower measured BMI.

[Insert Figure 2 about here.]

159 Age was not significantly correlated with female participants' reporting bias for height $160(r=0.11)$, body mass $(\mathrm{r}=-0.28)$ or BMI calculations $(\mathrm{r}=-0.34)$, but male participants' age was 161 significantly correlated with their reporting bias for height $(r=0.20, p=0.023)$ and BMI 162 calculations $(r=-0.17, p=0.049)$, but not for body mass $(r=-0.04)$. Older male participants 163 over-reported height and under-reported BMI more than their younger counterparts. Table 1 164 depicted the response bias comparisons between normal-weight and overweight male 
165 participants. There were no significant differences between normal and over-weight male

166 participants with respect to age, measured and self-reported height, and $\Delta$ height. Compared

167 to their normal-weight peers, over-weight participants had larger measured $\left(\mathrm{t}_{133}=-9.283\right.$,

$168 p<0.001, d=1.58)$ and self-reported body mass ( $\left.\mathrm{t}_{133}=-8.602, p<0.001, d=1.46\right)$, and greater

$169 \Delta$ body mass $\left(\mathrm{t}_{133}=-3.678, p<0.001, d=0.62\right)$, as well as larger measured BMI $\left(\mathrm{t}_{133}=-14.016\right.$,

$170 p<0.001, d=2.35)$ and self-reported BMI $\left(\mathrm{t}_{133}=-12.522, p<0.001, d=2.08\right)$, and greater $\Delta \mathrm{BMI}$

$171 \quad\left(\mathrm{t}_{133}=3.700, p<0.001, d=0.63\right)$.

172

[Insert Table 1 about here.]

173 
Main findings of the present study were that (a) recreational marathoners under-reported their body mass (i.e., they claimed that they were lighter than measurements revealed) leading to underestimated BMIs, and they over-reported their height (i.e., they claimed that they were taller than measurements revealed); (b) female marathon runners under-reported their body mass (and therefore our calculations of their BMI) more than did males; (c) male marathon runners with higher measured BMI under-reported their body mass (and therefore our calculations of their BMI) more than their counterparts with lower measured body mass; and (d) older male marathon runners over-reported their height and under-reported their body mass (and therefore our calculations of their BMI) more did than their younger counterparts. The observed self-report bias in these anthropometric characteristics was consistent with reports from previous research with different participant samples, confirming a general human tendency to overestimate height and underestimate body mass, frequently resulting in calculations that underestimate BMI (Basterra-Gortari, Bes-Rastrollo, Forga, Martínez, \& Martínez-González, 2007; Bes-Rastrollo, Pérez Valdivieso, Sánchez-Villegas, Alonso, \& Martínez-González, 2005; Bibiloni et al., 2016). The marathon runners' self-report bias for body mass $(\sim 0.6 \mathrm{~kg})$, BMI $\left(\sim 0.35 \mathrm{~kg} \cdot \mathrm{m}^{-2}\right)$ and height $(\sim 0.4 \mathrm{~cm})$ observed in the present study

191 was consistent with a previous study of athletes that reported an underestimate of BMI 192 calculations by $0.4 \mathrm{~kg} \cdot \mathrm{m}^{-2}$ (Knechtle et al., 2012). On the other hand, our marathon runners' 193 self-report bias was smaller than has been reported in the general population (Ikeda, 2016).

194 In addition, since BMI was related to marathon race time (Vickers \& Vertosick, 2016) and 195 runners' injury prevalence (Vitez et al., 2017), marathon runners may more frequently 196 monitor their body mass.

197 An analysis of $\operatorname{sex} \times$ assessment method interactions on these anthropometric 198 characteristics revealed that female marathon runners under-reported their body mass more 
than their male counterparts, also leading to females' under-reported BMI in our BMI

200 calculations based on their self-reported body mass. Our findings were also consistent with 201 the results of previous studies on different participant samples (Alvarez-Torices, Franch202 Nadal, Alvarez-Guisasola, Hernandez-Mejia, \& Cueto-Espinar, 1993; Bolton-Smith, 203 Woodward, Tunstall-Pedoe, \& Morrison, 2000; Roberts, 1995; Stunkard \& Albaum, 1981). 204 Under-reporting of body mass among female participants in these studies has been attributed 205 to women's particularly prevalent desire to be slim (Bolton-Smith et al., 2000; Giles \& 206 Hutchinson, 1991; Nichter \& Nichter, 1991). However, further investigation of the cause or 207 reason for this differential sex finding is needed. We also observed a self-report based BMI 208 bias among those male runners with higher self-report based BMI levels, although these BMI 209 calculation differences may have been largely influenced by differences in the participants' 210 self-reported height, rather than in self-reported body mass. This finding was based both on 211 the Bland-Altman plots analysis and the comparison between normal-weight and over-weight 212 male runners. The higher under-estimation of BMI using self-report measures in participants 213 with high BMI, compared to their counterparts with lower BMI, highlighted the need to 214 interpret such data with caution (Alvarez-Torices et al., 1993; Nieto-García et al., 1990). 215 With regards to the role of age in self-report bias, we found a larger bias among older 216 versus younger male marathon runners, consistent with prior findings in studies of athletes 217 (Knechtle et al., 2012) and non-athletes (Kuczmarski, Kuczmarski, \& Najjar, 2001). Knechtle 218 et al. (2012) showed that male (but not female) athletes $>35$ years old under-reported body 219 mass (and our calculations of their BMI) more than their younger counterparts. Kuczmarski 220 et al. (2001) found that self-report bias in BMI calculations among non-athletes increased 221 directly with age. Under-reporting BMI in older adults might be attributed partly to over222 reporting height, due to the lengthy time passage since height was last measured and/or 223 reduction that occurs in height with age (Stewart, Jackson, Ford, \& Beaglehole, 1987). 224 However, it is also clearly possible that this self-report bias emanates from a particularly 
225 prevalent male desire to be taller. As with speculations regarding women's particular self226 report bias toward under-estimating body mass, this possibility warrants further research.

227 Limitations of the present study include the lack of additional data to investigate or 228 delineate underlying reasons for sex-specific, age-specific, or BMI level-specific findings 229 regarding self-report bias. Additionally, our participants recorded their self-reported data 230 using a 'paper and pencil' questionnaire, introducing a methodological difference relative to 231 studies that used interviews or online tools to collect these data. Furthermore, the self-report 232 bias we observed may be contextually limited to the Greek culture ( $\mathrm{Ng}, 2019)$. The findings 233 of our study should be considered with caution given our sample size and should be verified 234 in future studies using larger samples. We acknowledge that our sample size ( $\mathrm{n}=167)$ was 235 smaller than that of studies participants in the general population (e.g. $n=272$, Arjunan et al., 236 2019). Additionally, our participants were aware that they were going to be measured for 237 actual height and body mass subsequent to completing questionnaires on which they self238 reported these variables; this knowledge may have influenced the degree of bias they 239 expressed, perhaps helping to explain a smaller bias in this sample than in studies with 240 participants in the general population. A strength of our study was its novel use of a sample 241 of recreational marathon runners who may be seen as falling between athletes and non242 athletes. Given the wide use of self-report measures to collect anthropometric data on 243 marathon runners (Ponzio et al., 2018; Vitez et al., 2017), our findings should be useful to 244 researchers and practitioners attempting to interpret data from these self-report assessment 245 methods.

\section{Conclusions}

247 Marathon runners under-reported body mass and calculated BMI, and over-reported 248 height, though the degree of disagreement between self-reported and measured 249 anthropometric data in the present sample was lower than has been observed in other studies, 
250 perhaps suggesting that marathon runners may have a more accurate self-perception or may 251 more accurately report their anthropometric characteristics compared to members of the 252 general population. According to our findings, self-reported body mass and height is apt to 253 be inaccurately reported by marathon runners, perhaps particularly for women with regard to 254 under reports of body mass and for older men with regard to over reports of height. The 255 present study was the first to examine the validity of such measures in recreational marathon 256 runners. 


\section{References}

259 Alvarez-Torices, J. C., Franch-Nadal, J., Alvarez-Guisasola, F., Hernandez-Mejia, R., \& 260 Cueto-Espinar, A. (1993). Self-reported height and weight and prevalence of obesity. 261 Study in a Spanish population. Int J Obes Relat Metab Disord, 17(11), 663-667.

262 Arjunan, P., Kovai, V., Jalaludin, B., \& Rooney, J. (2019). Validity of self-reported 263 anthropometrics in Arabic-speaking adults in Australia. Health Promot J Austr, in 264 print. doi: $10.1002 /$ hpja.313

265 Basterra-Gortari, F. J., Bes-Rastrollo, M., Forga, L., Martínez, J. A., \& Martínez-González, 266 M. A. (2007). Validity of self-reported body mass index in the National Health Survey. Anales del Sistema Sanitario de Navarra, 30(3), 373-381.

Bes-Rastrollo, M., Pérez Valdivieso, J. R., Sánchez-Villegas, A., Alonso, Á., \& MartínezGonzález, M. Á. (2005). Validation of self-reported weight and body mass index of the participants of a cohort of university graduates. Revista Espanola de Obesidad, $3(6), 352-358$.

Bibiloni, M. M., Coll, J. L. I., Salas, R., Pich, J., Pons, A., \& Tur, J. A. (2016). Ten-year trends (2000-2010) in bias of self-reported weight, height and body mass index in a Mediterranean adult population. Nutricion Hospitalaria, 33(6), 1367-1371. doi: $10.20960 /$ nh. 239

Bland, J. M., \& Altman, D. G. (1986). Statistical methods for assessing agreement between two methods of clinical measurement. Lancet, 1(8476), 307-310.

Boldt, P., Knechtle, B., Nikolaidis, P., Lechleitner, C., Wirnitzer, G., Leitzmann, C., . . . Wirnitzer, K. (2018). Quality of life of female and male vegetarian and vegan endurance runners compared to omnivores - results from the NURMI study (step 2). J Int Soc Sports Nutr, 15(1), 33. doi: 10.1186/s12970-018-0237-8

Bolton-Smith, C., Woodward, M., Tunstall-Pedoe, H., \& Morrison, C. (2000). Accuracy of the estimated prevalence of obesity from self reported height and weight in an adult Scottish population. Journal of Epidemiology and Community Health, 54(2), 143148. doi: 10.1136/jech.54.2.143 
Cohen, J. (1988). Statistical power analysis for the behavioral sciences (2nd ed.). Hillsdale, NJ: Lawrence Erlbaum Associates.

Connor Gorber, S., Tremblay, M., Moher, D., \& Gorber, B. (2007). A comparison of direct vs. self-report measures for assessing height, weight and body mass index: a systematic review. Obes Rev, 8(4), 307-326. doi: 10.1111/j.1467-789X.2007.00347.x

Giles, E., \& Hutchinson, D. L. (1991). Stature- and age-related bias in self-reported stature. J Forensic Sci, 36(3), 765-780.

Holmich, P., Christensen, S. W., Darre, E., Jahnsen, F., \& Hartvig, T. (1989). Non-elite marathon runners: health, training and injuries. Br J Sports Med, 23(3), 177-178. doi: 10.1136/bjsm.23.3.177

Ikeda, N. (2016). Validity of Self-Reports of Height and Weight among the General Adult Population in Japan: Findings from National Household Surveys, 1986. PLoS ONE, 11(2), e0148297-e0148297. doi: 10.1371/journal.pone.0148297

Knechtle, B., Rust, C. A., Rosemann, T., Knechtle, P., \& Bescos, R. (2012). Estimation bias: body mass and body height in endurance athletes. Percept Mot Skills, 115(3), 833844. doi: 10.2466/03.27.pms.115.6.833-844

Kuczmarski, M. F., Kuczmarski, R. J., \& Najjar, M. (2001). Effects of age on validity of selfreported height, weight, and body mass index: Findings from the third National Health and Nutrition Examination Survey, 1988-1994. Journal of the American Dietetic Association, 101(1), 28-34.

$\mathrm{Ng}$, C. D. (2019). Biases in self-reported height and weight measurements and their effects on modeling health outcomes. SSM Popul Health, 7, 100405. doi: 10.1016/j.ssmph.2019.100405

Nichter, M., \& Nichter, M. (1991). Hype and weight. Med Anthropol, 13(3), 249-284. doi: $10.1080 / 01459740.1991 .9966051$

Nieto-García, F. J., Bush, T. L., \& Keyl, P. M. (1990). Body mass definitions of obesity: Sensitivity and specificity using self-reported weight and height. Epidemiology, 1(2), 146-152. doi: 10.1097/00001648-199003000-00011 
314 Nikolaidis, P. T., Chalabaev, A., Rosemann, T., \& Knechtle, B. (2019). Motivation in the 315 athens classic marathon: The role of sex, age, and performance level in Greek 316 recreational marathon runners. International Journal of Environmental Research and Public Health, 16(14). doi: 10.3390/ijerph16142549

318 Nikolaidis, P. T., \& Knechtle, B. (2019). Force-velocity characteristics and maximal anaerobic power in male recreational marathon runners. Research in Sports Medicine. doi: $10.1080 / 15438627.2019 .1608993$

Nikolaidis, P. T., Rosemann, T., \& Knechtle, B. (2018a). Age-predicted maximal heart rate in recreational marathon runners: A cross-sectional study on Fox's and Tanaka's equations. Frontiers in Physiology, 9(MAR). doi: 10.3389/fphys.2018.00226

Ponzio, D. Y., Syed, U. A. M., Purcell, K., Cooper, A. M., Maltenfort, M., Shaner, J., \& Chen, A. F. (2018). Low Prevalence of Hip and Knee Arthritis in Active Marathon Nikolaidis, P. T., Rosemann, T., \& Knechtle, B. (2018b). Force-velocity characteristics, muscle strength, and flexibility in female recreational marathon runners. Frontiers in Physiology, 9(NOV). doi: 10.3389/fphys.2018.01563 Runners. J Bone Joint Surg Am, 100(2), 131-137. doi: 10.2106/jbjs.16.01071

Roberts, R. J. (1995). Can self-reported data accurately describe the prevalence of overweight? Public Health, 109(4), 275-284. doi: 10.1016/s0033-3506(95)80205-3

Seijo, M., Minckas, N., Cormick, G., Comandé, D., Ciapponi, A., \& BelizÁn, J. M. (2018). Comparison of self-reported and directly measured weight and height among women of reproductive age: a systematic review and meta-analysis. Acta Obstetricia et Gynecologica Scandinavica, 97(4), 429-439. doi: 10.1111/aogs.13326

Stewart, A. W., Jackson, R. T., Ford, M. A., \& Beaglehole, R. (1987). Underestimation of relative weight by use of self-reported height and weight. Am J Epidemiol, 125(1), 122-126. doi: 10.1093/oxfordjournals.aje.a114494

Stunkard, A. J., \& Albaum, J. M. (1981). The accuracy of self-reported weights. Am J Clin Nutr, 34(8), 1593-1599. doi: 10.1093/ajcn/34.8.1593

341 Vadeboncoeur, T. F., Silvers, S. M., Taylor, W. C., Shapiro, S. A., Roth, J. A., Diehl, N., . . 342 . Mohseni, M. M. (2012). Impact of a high body mass index on lower extremity injury 
in marathon/half-marathon participants. Journal of Physical Activity and Health, 9(1), 96-103. doi: 10.1123/jpah.9.1.96

345 Vickers, A. J., \& Vertosick, E. A. (2016). An empirical study of race times in recreational endurance runners. BMC Sports Sci Med Rehabil, 8(1), 26. doi: 10.1186/s13102-016$0052-\mathrm{y}$

348 Vitez, L., Zupet, P., Zadnik, V., \& Drobnic, M. (2017). Running Injuries in the Participants 349 of Ljubljana Marathon. Zdr Varst, 56(4), 196-202. doi: 10.1515/sjph-2017-0027

350 Vitti, A., Nikolaidis, P. T., Villiger, E., Onywera, V., \& Knechtle, B. (2019). The "New York 351 City Marathon": participation and performance trends of $1.2 \mathrm{M}$ runners during half$352 \quad$ century. Res Sports Med, 1-17. doi: 10.1080/15438627.2019.1586705 
Table 1. Self-reported and measured anthropometric characteristics.

\begin{tabular}{|c|c|c|}
\hline & Normal-weight $(n=82)$ & Overweight $(n=53)$ \\
\hline Age (years) & $43.2 \pm 8.3$ & $46.0 \pm 9.2$ \\
\hline \multicolumn{3}{|l|}{ Height } \\
\hline Measured height $(\mathrm{cm})$ & $176.6 \pm 5.9$ & $175.6 \pm 5.9$ \\
\hline Self-reported height $(\mathrm{cm})$ & $176.9 \pm 5.6$ & $176.3 \pm 5.9$ \\
\hline$\Delta$ height (cm) & $0.2 \pm 1.3$ & $0.6 \pm 1.5$ \\
\hline \multicolumn{3}{|l|}{ Weight } \\
\hline Measured weight (kg) & $72.2 \pm 6.3$ & $84.2 \pm 8.6^{*}$ \\
\hline Self-reported weight $(\mathrm{kg})$ & $72.0 \pm 6.2$ & $83.2 \pm 8.9^{*}$ \\
\hline$\Delta$ weight (kg) & $-0.2 \pm 1.0$ & $-1.0 \pm 1.4^{*}$ \\
\hline \multicolumn{3}{|l|}{ BMI } \\
\hline Measured BMI (kg.m-2) & $23.1 \pm 1.3$ & $27.3 \pm 2.1^{*}$ \\
\hline $\begin{array}{l}\text { Self-reported BMI (kg.m } \\
\left.{ }^{2}\right)\end{array}$ & $23.0 \pm 1.3$ & $26.7 \pm 2.2 *$ \\
\hline$\Delta B M I\left(\mathrm{~kg} \cdot \mathrm{m}^{-2}\right)$ & $-0.1 \pm 0.5$ & $-0.5 \pm 0.7^{*}$ \\
\hline
\end{tabular}




\section{Legends of figures}

371

372

373 374

376

377

378

379

380

381

382

383

384

385

386

387

388

Figure 1. Variation of height, body mass and body mass index by assessment method and sex.

BMI=body mass index. * main effect of assessment method on variable at $\mathrm{p}<0.05$. \# sex $\times$ assessment method interaction on variable at $\mathrm{p}<0.05$.

Figure 2. Bland-Altman plots showing the difference (bias) between reported and measured height, weight and body mass index.

BMI=body mass index; Difference=reported-measured; Average $=($ reported + measured $) / 2$. 
Height

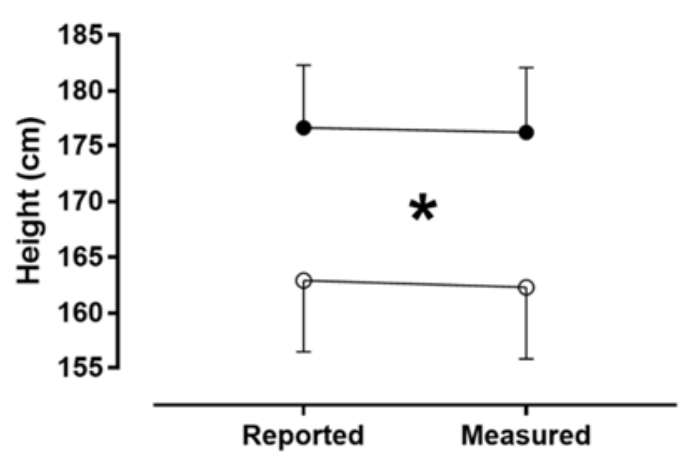

Assessment method
Body mass

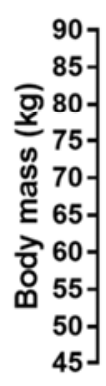

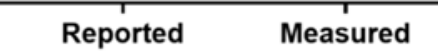

Assessment method
BMI

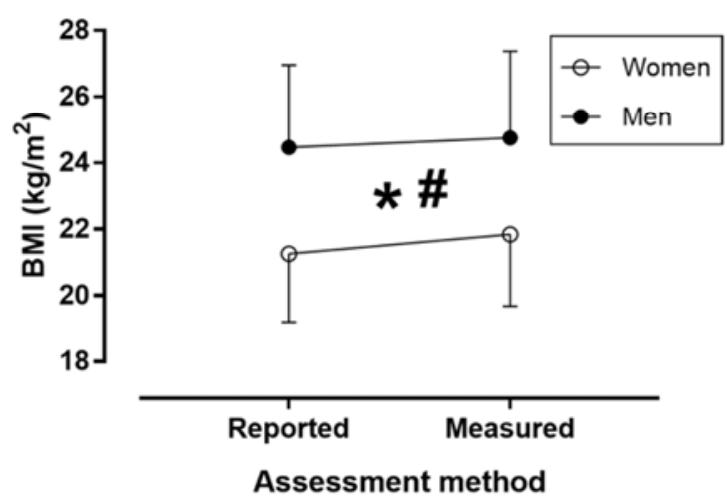

$390 \quad$ Figure 1 


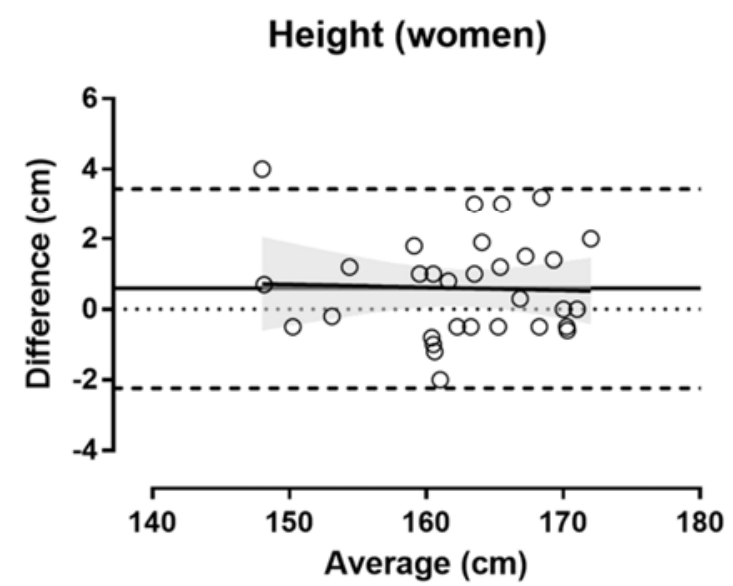

Height (men)

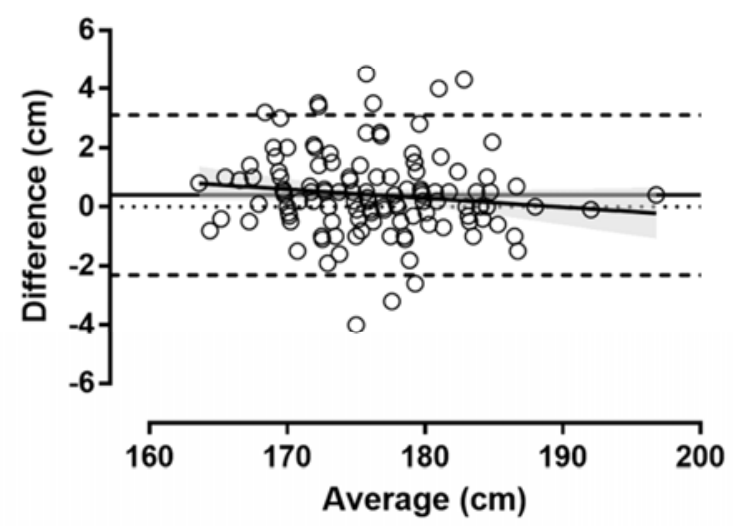

Figure 2
Body mass (women)

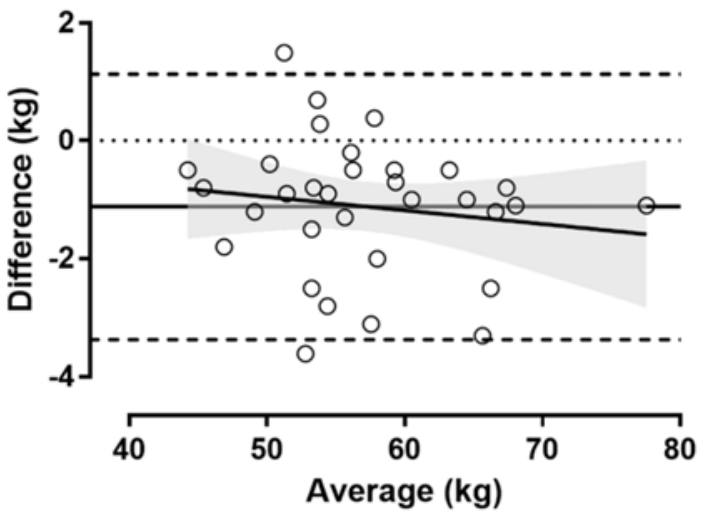

Body mass (men)

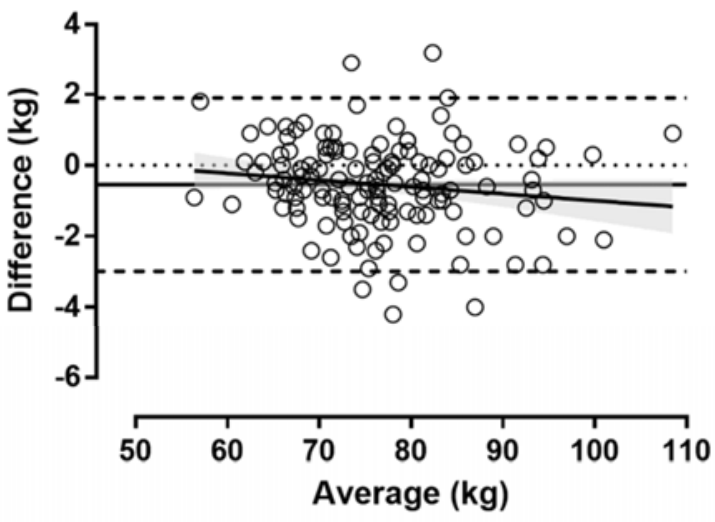

BMI (women)
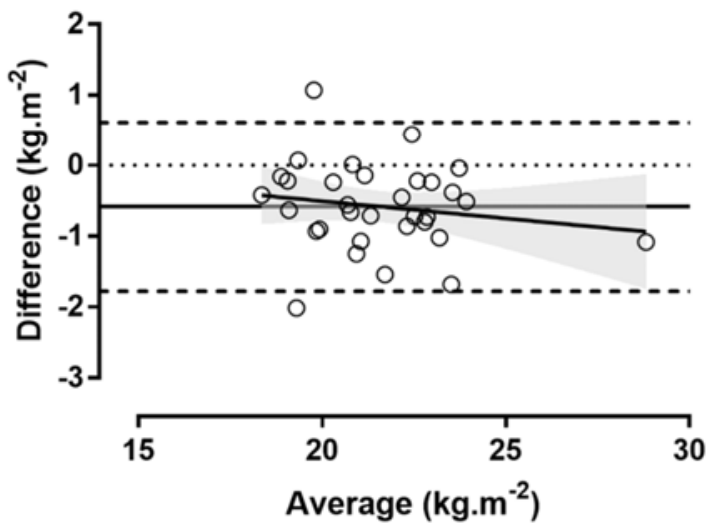

BMI (men)

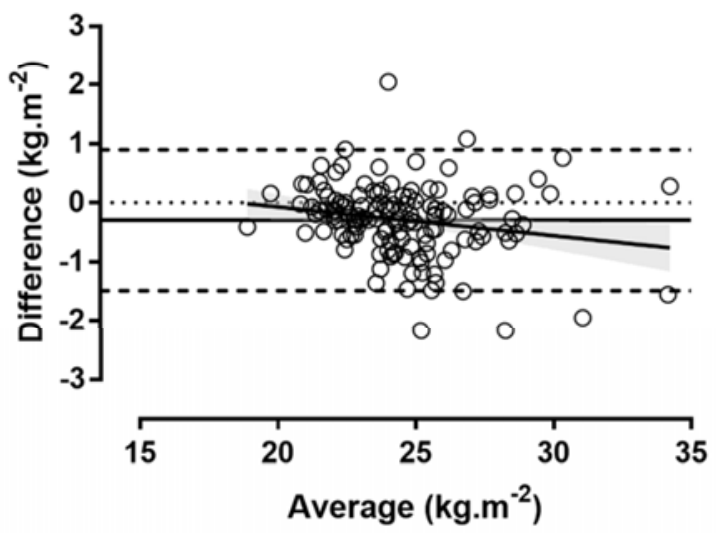

